See discussions, stats, and author profiles for this publication at: https://www.researchgate.net/publication/1781028

\title{
Classical and quantum decay of one dimensional finite wells with oscillating walls
}

Article in Physical review. E, Statistical physics, plasmas, fluids, and related interdisciplinary topics · February 1997

DOI: 10.1103/PhysRevE.55.6507 · Source: arXiv

CITATIONS

3

2 authors:

Alejandro Fendrik

Universidad Nacional de General Sarmiento

41 PUBLICATIONS 267 CITATIONS

SEE PROFILE

Some of the authors of this publication are also working on these related projects:

Project complex biological systems View project
READS

19

D. A. Wisniacki

University of Buenos Aires

80 PUBLICATIONS 814 CITATIONS

SEE PROFILE 


\title{
Classical and quantum decay of one dimensional finite wells with oscillating walls.
}

\author{
A.J.Fendrik日 and D.A.Wisniacki讯 \\ Departamento de Física, Facultad de Ciencias Exactas y Naturales, \\ Universidad de Buenos Aires, Ciudad Universitaria, 1428, Buenos Aires, \\ Argentina.
}

(February 5, 2008)

\begin{abstract}
To study the time decay laws (tdl) of quasibounded hamiltonian systems we have considered two finite potential wells with oscillating walls filled by non interacting particles. We show that the tdl can be qualitatively different for different movement of the oscillating wall at classical level according to the characteristic of trapped periodic orbits. However, the quantum dynamics do not show such differences.

$05.45 .+\mathrm{b}$
\end{abstract}

Typeset using REVTEX 


\section{INTRODUCTION}

The time decay laws (tdl) of classical quasibounded hamiltonian systems use to include meaningful information related to the bounded transient dynamics. The feature of the tdl corresponding to a fully chaotic system has been well established. In a special way, the tdl's are greatly sensible to the characteristic of the trapped periodic orbits. Systems having a fully hyperbolic set of trapped periodic orbits show a purely exponential tdl, while the

tdl becomes algebraic if they have a parabolic subset of them. So, in the case of the Sinai billiard [1] and the Bunimovich stadium [2], paradigms of fully chaotic hamiltonian systems, the existence of the so called bouncing ball orbits (parabolic and non isolated periodic orbits) gives algebraic long time tails in the tdl's [3] 5$]$.

On the other hand, it is a well known fact that the characteristic and the distribution of periodic orbits in bounded systems are relevant subjects to quantify semiclassically such systems [6].

To establish the influence of the periodic orbits in the decay of quantum systems, in the present work we have studied the decay processes in two quasibounded systems whose trapped periodic orbits have different characteristics.

We show that the classical systems having trapped periodic orbits of different characteristics have qualitatively different tdl, while the quantum analogues do not show such sensitivity. In other words, we show that two classical systems with exponential and algebraic tdl respectively they have algebraic quantum tdl (qtdl) when they are considered as quantum systems.

Our work is organized in the following way. In Sec. II we introduce the classical systems whose tdl we study in Sec. III. Sec. IV is devoted to describe the quantum systems and the numerical method that we have employed to compute the qtdl. In Sec. $\nabla$ we show the resulting qtdl. Finally, Sec. V1 is devoted to conclusions. We include one appendix to show some characteristics of the tdl's. 


\section{THE CLASSICAL SYSTEMS}

In the present work we deal with a kind of one-dimensional periodically driven hamiltonians. The corresponding system can be described in the extended phase space [7] as a conservative system with two degrees of freedom.

Let us consider non interacting point particles of unity mass moving inside a onedimensional time dependent potential well

$$
V(q, t)=\left\{\begin{array}{l}
\infty \text { if } q<0, \\
0 \quad \text { if } 0 \leq q \leq a(t) \\
\infty \text { if } a(t)<q .
\end{array}\right.
$$

That is a perfectly reflecting one-dimensional box with an oscillating wall according to the time dependent law $a(t)$.

Looking at the graph of the position of one particle $q$, as a function of time $t$, we can see that the system is equivalent to a particle forwards in a two dimensional infinite pipe with appropriate reflecting conditions at the boundary (See Fig. [(a)) . The reflecting condition (the change of sign of the relative velocity between the walls and the particle) imposes:

$$
\begin{aligned}
& v_{f}=-v_{i} \quad \text { at } q=0, \\
& v_{f}=2 \dot{a}(t)-v_{i} \quad \text { at } q=a(t),
\end{aligned}
$$

where $v_{f}\left(v_{i}\right)$ is the velocity of the particle after (before) the collision. Both velocities correspond to the sloops of the straight lines in the q.vs.t graphs $(v=\tan \alpha)$.

Let be $a(t)$ periodic in time, so $a(t)=a(t+\tau)$.

Taking account the time lattice translation symmetry we can consider only an elemental segment of the channel setting periodic boundary conditions, namely $t=\tau \rightarrow t=0$ so that $q(t=\tau) \rightarrow q(t=0)$ and $v(t=\tau) \rightarrow v(t=0)$. So, the system can be seen as a two dimensional billiard where particles leaving at $t=\tau$ emerge at $t=0$ having the same velocity $v$ and the same position $q$ while the collisions at the walls follows the laws (2.2). In this version of the system, we have two degrees of freedom associated to the coordinates $q$ 
and $\theta=t-[t / \tau] \tau$ (reduced time) where [...] means the integer part (See Fig. 1 (b)). The conjugate momenta are $v$ and $-E$ (kinetic energy) respectively.

When the potential well is given by rule (2.1) particles inside the box are bounded despite their velocities. On the other hand, if the oscillating wall (at $q=a(t)$ ) is a finite barrier of potential of height $V_{0}$, particles with velocities $|v|>\sqrt{2 V_{0}}$ will leave the well when they reach the wall. In this case, according to the characteristic of the dynamics, which is controlled by the law $a(t)$ of the oscillating wall, the system can be transiently bounded.

We have focused our attention in two particular rules $a(t)$. In the following we take $\tau$ as a unit of time and the mean value of $a(t),<a>_{t}=1 / \tau \int_{0}^{\tau} a(t) d t$ as a unit of length and we take the energies as dimensionless (that is they are divided by $m<a>_{t}^{2} / \tau^{2}$ ).

We have considered:

a) Harmonic oscillations

That is to say:

$$
a(t)=1+\delta \sin 2 \pi t
$$

This system resembles the so called full Fermi accelerator model. Such system was widely studied in simplified versions [7]. Figure \& shows the Poincare surface section for $q=0$ corresponding to the kinetic energy $E$ versus the $\theta$. Here we can distinguish three regions. The low energy region is fully chaotic, the intermediate energy region shows mixed dynamics (regular island surrounded by the chaotic sea) and finally the adiabatic region, where the dynamics become regular and we can see invariant curves.

Now we assume that the moving wall is a finite barrier of potential of height $V_{0}$. So, the particles can leave the well and the limit between the bounded motion and the unbounded one is the line $E=V_{0}$.

If $V_{0}$ lies on the adiabatic region, that is there is an invariant curve below the limit line, the system will remain bounded. So to study the tdl we will set $V_{0}$ less than the lower invariant curve.

b) Sawtooth oscillations 
In this case:

$$
a(t)= \begin{cases}(1+4 \delta t) & \text { if } 0 \leq t \leq 1 / 4, \\ (1+2 \delta)-4 \delta t & \text { if } 1 / 4<t \leq 3 / 4, \\ (1-4 \delta)+4 \delta t & \text { if } 3 / 4<t<1 .\end{cases}
$$

The main feature of this system is that the periodic orbits are parabolic and non isolated. The two dimensional equivalent billiard is like billiards with neutral boundaries as polygonal ones [8,9]. For example, Fig. 3 shows a representative periodic orbit. Looking at the billiard as map $\mathcal{T}$

$$
(q(\theta=0), \tan \alpha(\theta=0)) \stackrel{\mathcal{I}}{\longrightarrow}\left(q^{\prime}(1), \tan \alpha^{\prime}(t=1)\right)
$$

the periodic orbit corresponds to a fixed point of $\mathcal{T}^{2}$. A straightforward calculation shows that $\mathcal{T}^{2}$ has eigenvalues $\lambda_{1}=\lambda_{2}=1$ when it is linearized at this fixed point. So, it corresponds to a parabolic periodic orbit. On the other hand, given a fixed point $\left(q_{0}, \tan \alpha_{0}\right)$, it can be shown that there exists an interval of $q, I=\left(q_{\min }, q_{\max }\right)$, such that $\left(q, \tan \alpha_{0}\right)$ is a fixed point if $q \in I$. That is, the fixed points are non isolated.

\section{THE DECAY OF CLASSICAL SYSTEMS}

To study the tdl, we fill a portion of the bounded region with $N_{0}=10^{5}$ particles whose initial conditions are uniformly (random) distributed in $q$ and $v=\tan \alpha$. We compute the ratio $N(t) / N_{0}$ of the remaining population as function of time.

Let us remark that the initial population must be chosen with low energy (in the chaotic region) to ensure the decay. If not so, particles can have initial conditions such that they will remain trapped because, in the case of harmonic oscillations, they can be on regular island included in the bounded region. On the other hand, we want to exclude the population of the region corresponding to very low energy to minimize the effect of the asymptotic orbits to the (trivial) parabolic subset of periodic orbits characterized by $E=0$. These trajectories are parallel lines to the time axis in the two dimensional pipe version of the system and they 
correspond to particles that having $v=0$, they will never hit the boundary of the well (see appendix). So we have considered

$$
E_{\min } \leq E \leq E_{\max }
$$

Figure 1 shows a part of the Poincare surface section of Fig. 20 and the region that was initially populated. We have taken $E_{\min }=2.64$ and $E_{\max }=5.35$.

Figures 5 (a) and (b) show the results corresponding to the harmonic and sawtooth oscillations in log-lin and log-log plot respectively. We can see that the curves are quite different. In the case of Fig. 5(a) the log-lin plot shows an almost exponential behavior for the harmonic oscillations while Fig. 5(b) the log-log plot shows an algebraic one for

the sawtooth oscillations. Such a difference can be explain in terms of the features of the periodic orbits that are included in the trapped region [3]. In the case of harmonic oscillations, the trapped periodic orbits are hyperbolic and isolated (aside the subset $E=0$ that was not initially populated) while they are parabolic and non-isolated (like the bouncing ball orbits of the Sinai billiard) for the sawtooth oscillations. We stress that the deviation of the exponential behavior in the case of harmonic oscillations can be attributed to the population of very low energy region by indirect way (after collisions, see appendix).

\section{QUANTUM SYSTEMS}

To study the quantum analogues of the systems, we want to solve the time dependent Schroedinger equation.

$$
i \hbar \frac{\partial \mid \alpha, t_{0}, t>}{\partial t}=\hat{H}(q, t) \mid \alpha, t_{0}, t>
$$

being $\mid \alpha, t_{0}, t_{0}>$ an initial condition and $\hat{H}(q, t)$ the Hamiltonian operator $\hat{H}=\hat{T}(\hat{p})+\hat{V}(\hat{q}, t)$ where:

$$
V(q, t)=\left\{\begin{array}{l}
0 \quad \text { if } 0 \leq q \leq a(t) \\
V_{0} \text { if } a(t)<q
\end{array}\right.
$$


We have employed an expectral method (fast Fourier transform, FFT) based on the split of the evolution operator $\hat{U}\left(t, t_{0}\right)$ [10 defined by

$$
\left|\alpha, t>=\hat{U}\left(t, t_{0}\right)\right| \alpha, t_{0}>
$$

This technique was widely applied to solve bounded systems [11,12], and transmission phenomena through moving barriers [13].

Such procedure does not avail with infinite barriers of potential, so to avoid the infinite barrier at $q=0$, we have employed the symmetrized potential

$$
V(q, t)= \begin{cases}0 & \text { if } 0<|q| \leq a(t) \\ V_{0} & \text { if } a(t)<|q|\end{cases}
$$

instead of potential (4.2).

We want to compute

$$
P_{\alpha}(t)=\int_{-a(t)}^{a(t)}\left|<\alpha, t_{0}, t\right| q>\left.\right|^{2} d q
$$

that is the probability to detect the particle inside the well at time $t$.

Because the system can be unbounded we will have a non stationary leaving flux of probability going to $q= \pm \infty$. The spectral method automatically imposes periodic boundary conditions, so the flux of probability leaving at $q=+(-) q_{\max }$ will appear as coming at $q=-(+) q_{\max }$. So we need to kill the leaving flux. To get this we have employed absorbing boundary conditions [14] adding an absorptive (purely imaginary) static potential barrier

$$
V_{A}=\frac{i U_{0}}{\cosh ^{2}\left(\gamma\left(q-q_{\text {max }}\right)\right)}+\frac{i U_{0}}{\cosh ^{2}\left(\gamma\left(q+q_{\text {max }}\right)\right)}
$$

to the actual (real) time dependent potential well 4.4. Here, $U_{0}$ and $\gamma$ are real parameters whose values were chosen to minimize the reflection and transmission coefficients of $V_{A}$. All computations were performed taking a time step $\Delta t=0.00064$ on a grid of 4096 steps of spatial sampling $\Delta q=0.00125$. The absorbing region at the boundaries includes 250 spatial steps. This fact determines the value of $\gamma$. We have taken $U_{0}=14.79$. Figures 6 summarizes the characteristic of the potential for the present calculation. 
On the other hand we have change the sharp step function in the potential 4.4 by a soft version like a Saxon-Woods profile

$$
V(q, t)=\frac{V_{0}}{1+\exp b\left(a(t)^{2}-q^{2}\right)}
$$

as was prescribed in Ref. [12] to improve the convergence of the numerical method. We have

found that to ensure convergence and stability, we need that the potential vary from $V_{0} / 10$ to $9 V_{0} / 10$ in two spatial steps $\Delta q$. This determines the parameter $b$.

\section{THE DECAY OF QUANTUM SYSTEMS}

To populate the quantum system in an equivalent way as in the classical calculation, we consider the evolution of a quantum ensemble given by

$$
\hat{\rho}\left(t_{0}\right)=\frac{1}{N} \sum_{\alpha \in N}\left|\alpha, t_{0}, t_{0}><\alpha, t_{0}, t_{0}\right|
$$

where $N$ is the number of eigenstates of the static well of deep $V_{0}=V(q, t=0)$ whose eigenenergies lie on the region that we have populated the classical system. Such number depends on the value of $\hbar$. Setting an appropriate value we calculate

$$
P(t) \equiv \int_{-a(t)}^{a(t)}<q|\hat{\rho}(t)| q>d q=\frac{1}{N} \sum_{\alpha \in N} P_{\alpha}(t) .
$$

We have compute (5.2) taking $\hbar=0.0255$. For this value there are ten bounded eigenstates of the static well whose eigenenergies are such that $E_{\min } \leq E \leq E_{\max }$.

The results are shown in Figure 7 a) and b). The first shows a log-lin plot where we can see that both curves, corresponding to harmonic oscillations (curve A) and sawtooth oscillations (curve B) have non-exponential behavior. The second one shows a log-log plot that evidences an algebraic law from $t>25$ for both curves unlike to the classical behaviors where for the same time interval they are qualitatively different (see Fig. 5(a) and (b)). Let us remark that even algebraic $\left(\propto 1 / t^{\beta}\right)$, the characteristic exponents of the qtdl are

quite different. The best fit for the qtdl corresponding to the harmonic oscillation system is $\beta=1.45$ while it is $\beta=1.11$ for the sawtooth oscillation system. 


\section{SUMMARY AND CONCLUSIONS}

We have studied the time decay laws of two quasibounded systems in the framework of classical and quantum mechanics. The classical results show that the tdl's of the two systems are qualitatively different. One of the tdl results exponential while the other is algebraic. This dissimilitude can be explain in term of the diverse characteristics of the trapped periodic orbits in the systems. On the other hand, the quantum systems do not show such difference. We have obtained algebraic qtdl for both systems. However, the characteristic exponents of the qtdl are different.

There are evidences that open quantum systems whose classical counterparts are chaotic while they remain bounded (with tdl almost exponential) can originate algebraic qtdl [15] with different characteristic exponents. This effect can be explained by the distribution (Gaussian) of resonances, assuming an exponential decay of each resonance and an initial population of them. Varying the initial population, different characteristic exponents can be obtained. Such algebraic decay does not seem to be related to the existence of parabolic periodic orbits that generate algebraic tdl in classical systems [0]. In our systems, the initial population is the same for both systems so we think that the different exponents can be originate by the distribution of antiresonances (that is particular states that are refractory to absorb energy from the oscillating wall) into each well.

On the other hand, in Ref. [16,17] it is shown that, in general, the qtdl corresponding to chaotic systems have a power long time tail $\left(\propto 1 / t^{\beta}\right)$. The specific characteristic exponent $\beta$, depends on the number of open channels for the decay. In particular, for one open channel the exponent is $\beta=1.5[18]$. This fact, could be related to our result $\beta=1.45$ for the harmonic oscillating wall system. Moreover, the characteristic exponent $\beta$, corresponding to non-chaotic systems for one open channel and strong coupling between quasibounded states and the continuum is closer to $\beta=1$ [19 because the widening of the distribution of resonance widths respect to those corresponding to chaotic systems. This result could be related to our result $\beta=1.11$ in the case of sawtooth oscillating wall system. These 
apparent connections are been studied.

To finish, in spite of the classical systems and the quantum systems considered in the present work are not completely analogues (essentially because the smoothing of the quantum potential), we expect that the tdl corresponding to the classical systems with the smoothing potential will be the same as the step potential systems. As we remark in Sec. III, the tdl for the classical systems is determined by the linear stability of the trapped periodic orbits and such characteristic seems to be related to the time dependence (instead the spatial dependence) of the potential.

\section{ACKNOWLEDGMENTS}

This work was partially supported by UBACYT(Ex079). We would like to thank E.Vergini for useful suggestions.

\section{APPENDIX A:}

The present appendix is devoted to show the effect in the tdl when the very low energy region is initially populated.

Let us consider a well of deep $V_{0}$. We have calculate the tdl starting off the uniform population of whole trapped region (that is $0 \leq q \leq 1$ and $-\sqrt{2 V_{0}} \leq v \leq \sqrt{2 V_{0}}$. Fig. 9(a) shows the tdl as a function of time. Here, we can see an algebraic behavior, that is

$$
N(t) / N_{0} \propto 1 / t^{\beta}
$$

where $\beta=1$.

According Ref. [3], we expect a subset of periodic parabolic orbits included in the bounded region. This subset corresponds to particles that having $v \approx 0$ generate trajectories which are asymptotic to the horizontal paths $v=0$ in the pipe version of our system.

To show this, let us consider $n(t)$ the fraction of initial conditions that spend a time $t^{\prime}$ greater than a given time $t$, to reach the oscillating wall. Looking at Fig. 8, we estimate, 
for $t>2 / \sqrt{2 V_{0}}$

$$
n(t)=\frac{1}{2 \sqrt{2 V_{0}}} \int d q \int_{-\tan \phi_{\min }=(1+q) / t}^{\tan \phi_{\max }=(1-q) / t} d(\tan \phi)
$$

So that

$$
n(t)=\frac{1}{\sqrt{2 V_{0}}} \times \frac{1}{t}
$$

Following Ref. [1], we assume for the long time tail

$$
N(t) / N_{0} \approx \omega \int_{t^{\prime}=t}^{t^{\prime}=\infty} g\left(t^{\prime}\right) d t^{\prime}
$$

where $g(t) d t$ is the fraction of initial conditions for the first collision with the moving wall occurs between $t$ and $t+d t$ and $\omega$ is the probability to leave the wall after one collision. As

$$
g(t) \approx \frac{-d n}{d t}
$$

we have the law A1 for the tdl. We stress that in the case of Fig. A1 the algebraic tail becomes for $t \approx 2 / \sqrt{2 V_{0}}$ but in the general, if we populate the low energy region such that $|v|<\left|v_{\max }\right|<\sqrt{2 V_{0}}$ we expect that the tdl exhibits a crossover between a stretched exponential and the algebraic decay law A1 for long times as it shows in Fig. 9(b).

When the initial population excludes the very low energy region, that is $\left|v_{\text {min }}\right| \leq|v| \leq$ $\left|v_{\max }\right|$, we have numerical evidence that the algebraic tail for long times follows $1 / t^{2}$ (See Fig. 10). So, it differs by one respect to the precedent. This difference also occurs in the decay of other quasibounded hamiltonian systems when a parabolic region is initially populated or it is populated by an indirect way in [20,21. 


\section{REFERENCES}

* Member of CONICET

** Fellowship UBA

[1] Ya.G.Sinai, Russ. Math. Surveys, 25, 137, (1970).

[2] L.A.Bunimovich, Func. Anal. Appl. 8, 254, (1974).

[3] A.J.Fendrik, A.F.M.Rivas and M.J.Sánchez, Phys. Rev. E 50, 1948, (1994).

[4] A.J.Fendrik and M.J.Sánchez, Phys. Rev. E 51, 2996, (1995).

[5] H.Alt, H.D.Gräf, H.L.Harney, R.Hofferbert, H.Rehfeld, A.Richter and P.Schardt, Phys. Rev. E 53, 2217, (1996).

[6] M.C.Gutzwiller, Chaos in Classical and Quantum Mechanics (Springer-Verlag, NY, 1990).

[7] A.J.Lichtenberg and M.A.Lieberman, Regular and Chaotic Dynamics (Springer-Verlag, Berlin, 1992).

[8] P.J.Richens and M.V.Berry, Physica D 2, 495, (1981).

[9] J.L.Vega, T.Uzer and J.Ford ,Phys. Rev. E 48, 3414, (1993).

[10] M.D.Feit, J.A.Fleck and A.Steiger, J. Comput. Phys. 47, 412 (1983).

[11] M.D.Feit, J.A.Fleck and A.Steiger, J. Chem. Phys. 78 , 1 (1988).

[12] S.Tomsovic and E.J.Heller, Phys. Rev. E 47, 282,, (1993).

[13] A.Pimpale, S.Holloway and R.J.Smith, J. Phys. A 24, 3533 (1991).

[14] R.Kosloff and D.Kosloff, J. Comput. Phys. 63, 363 (1986).

[15] H.Alt, H.D.Gräf, H.L.Harney, R. Hofferbert, H.Lengeler, A.Richter, P. Schardt and H.A.Weidenmüller, Phys. Rev. Lett. 74, 62, (1995). 
[16] C.H.Lewenkopf and H.A.Weidenmüeller, Ann. Phys. (N.Y.) 212, 53, (1991).

[17] H.L.Harney, F.M.Dittes and A.Müeller, Ann. Phys. (N.Y.) 220, 159, (1992).

[18] F.M.Dittes, H.L.Harney and A.Mueller, Phys. Rev. A 45, 701, (1992).

[19] F.M.Dittes, I.Rotter and T.H.Seligman, Phys. Lett. A 158, 14, (1991).

[20] A.S.Pikovsky, J. Phys. A 25, L477, (1992).

[21] A.J.Fendrik and M.J.Sánchez, J. Phys. A 28, 4235 (1995). 


\section{FIGURES}

FIG. 1. a) One trajectory in the two dimensional pipe version of the system. The coordinates are the time $t$ and the position of the particle $q$. b) The same trajectory in the billiard version. This point of view is obtained by exploting the time lattice translation symmetry of the pipe setting $\theta=t-[t / \tau] \tau$ as a coordinate.

FIG. 2. $E$ (kinetic energy) vs. $\Theta$ (reduced time) at $q=0$. Poincare surface section corresponding to the system when the wall at $q=a(t)$ oscillates armonically. Both, $E$ and $\Theta$ are dimensionless and the amplitude of the oscillation is $\delta=0.2$ (in units of $\langle a\rangle_{t}$, see the text).

FIG. 3. One parabolic periodic orbit corresponding to the sawtooth oscillation. Here $q$ is the position of the particle while $\theta$ is the reduced time.

FIG. 4. $E$ (kinetic energy) vs. $\Theta$ at $q=0$. This Poincare surface section shows only the low energy region and it was generated by one initial condition. The solid straight lines limit the initial population that is mentioned in the text. The dashed line indicates the deep of the well (it separates the bounded region and the unbounded region). $E$ and $\Theta$ are dimensionless.

FIG. 5. a) Log-lin plot of the remaining population $N / N_{0}$ inside the well against $t$ (tdl). We have fixed $\tau$ (the period of the moving wall) as the unit of time and $\langle a\rangle_{t}$ (the mean value of the position of the moving wall) as the unit of length. (A) correspond to the harmonic oscillations system and it has an almost exponential behavior while (B) correspond to the sawtooth oscillations. In both cases the amplitude of the oscillation is $\delta=0.2$ and the deep of the well is $V_{0}=8.63$. b) The same curves of a) but in a log-log plot to show the algebraic behavior of (B).

FIG. 6. This Figure shows the characteristic of the potential for the computation of qtdl. The spatial grid has 4096 steps. The absorbing region is marked by the shadow rectangles at the boundaries. Each side has 250 steps. 
FIG. 7. a) Log-lin plot corresponding to the qtdl's $P$ vs. $t$. Curve (A) corresponds to harmonic oscillation. Curve (B) corresponds to sawtooth oscillations. b) Log-log plot for the same laws for $t>25$ to show the algebraic behavior. The units are the same as Figure 5 .

FIG. 8. Graph to show the fraction of initial conditions for which the first collision on the moving wall occurs in times greater than $t$. Given an initial position $q$, the velocities are $v=\tan \phi$ such $-\phi_{\min } \leq \phi \leq \phi_{\max }$.

FIG. 9. a) Log-log plot of the tdl corresponding to harmonic oscillating well system starting off the uniform population of whole trapped region. The deep of the well is $V_{0}=0.78$ and $\delta=0.2$. We have also drawn the straight line of slope one to clarify the characteristic exponent of the algebraic behavior. b)Log-log plot of the tdl corresponding to harmonic oscillating wall. In this case $V_{0}=19.7, \delta=0.2$ and the initial population is the same of a), that is $|v| \leq 1.25$. Here we can see the algebraic behavior for the long time tail. We also draw the straight line of slope one. The unit of time and the unit of length are the same as in Figure 5 .

FIG. 10. Log-log plot of the tdl corresponding to harmonic oscillating wall. In this case $V_{0}=8.63, \delta=0.2$ and the initial population was uniform in the region shown in the Figure 4 which excludes the parabolic region. Here we can see the algebraic behavior for the long time tail. We also draw the straight line of slope 2. The unit of time and the unit of length are the same as in Figure 5 . 


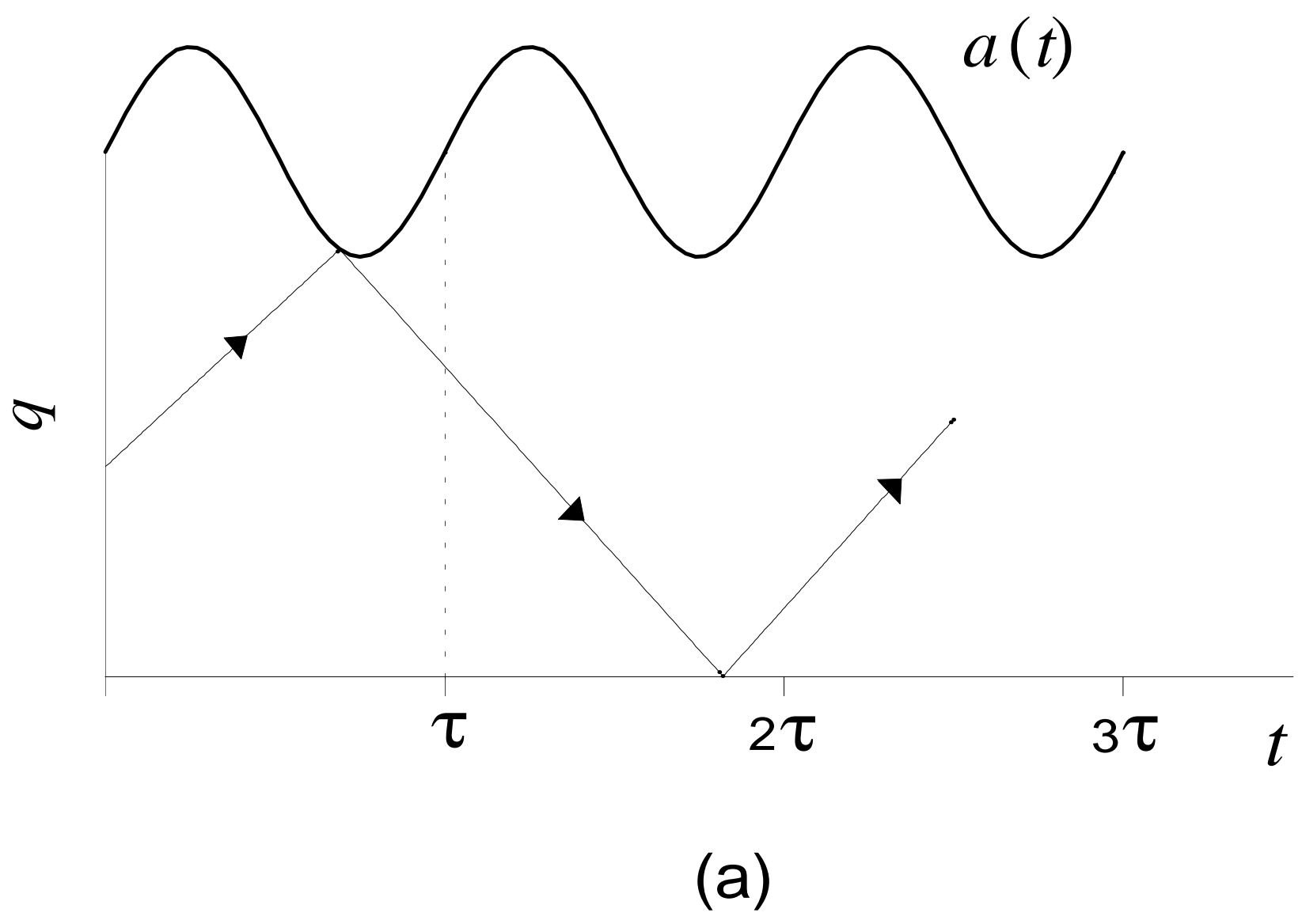




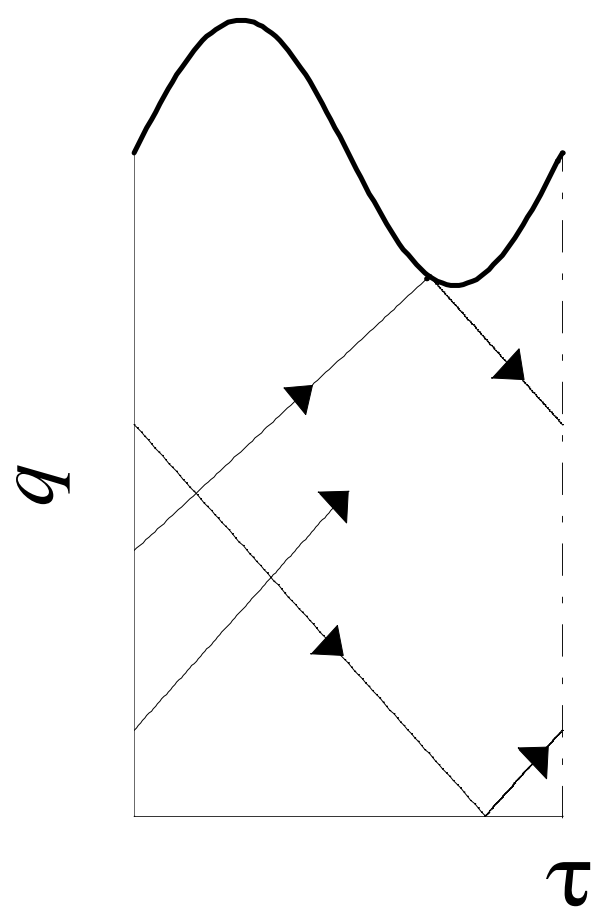

(b) 


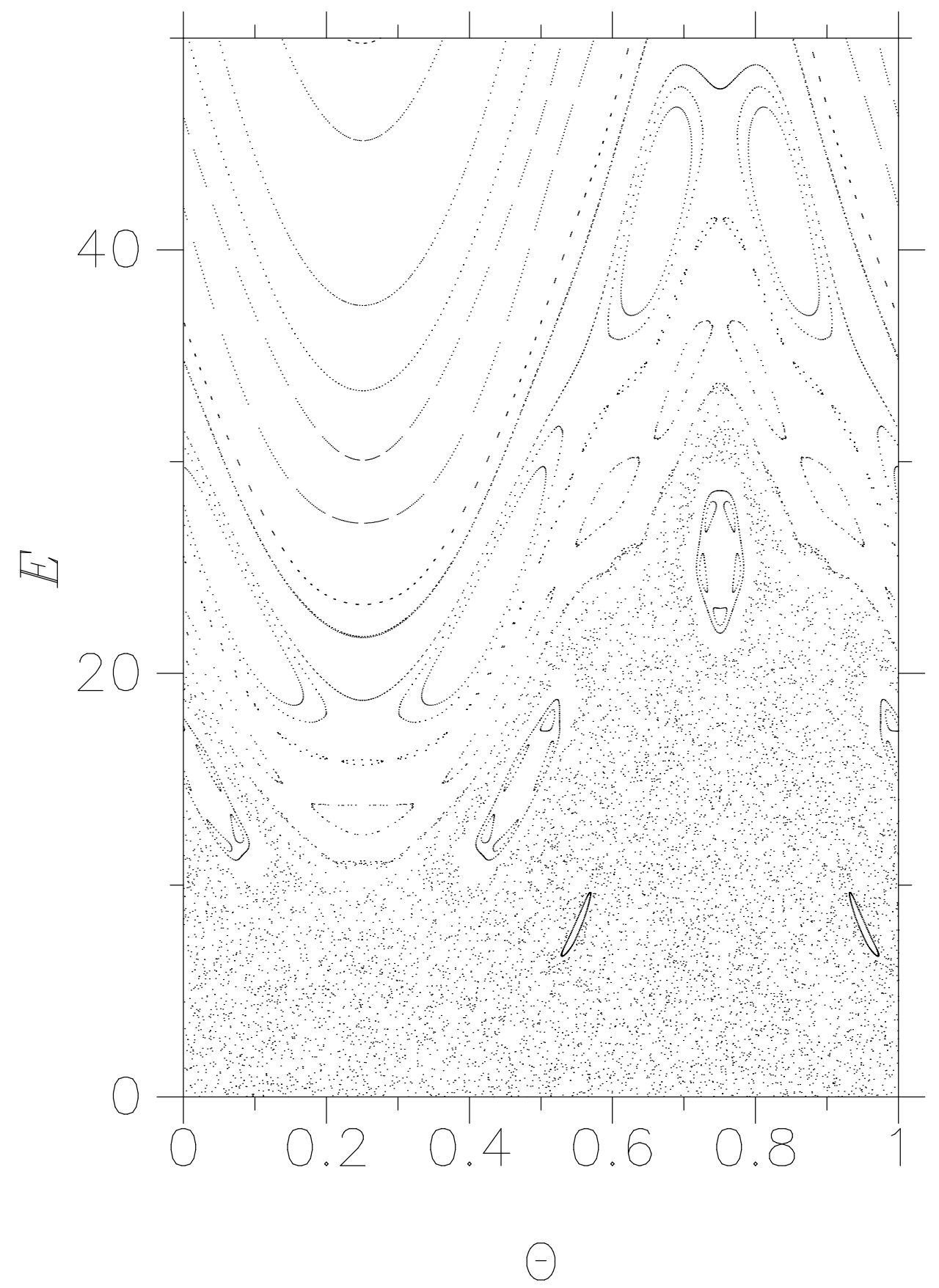




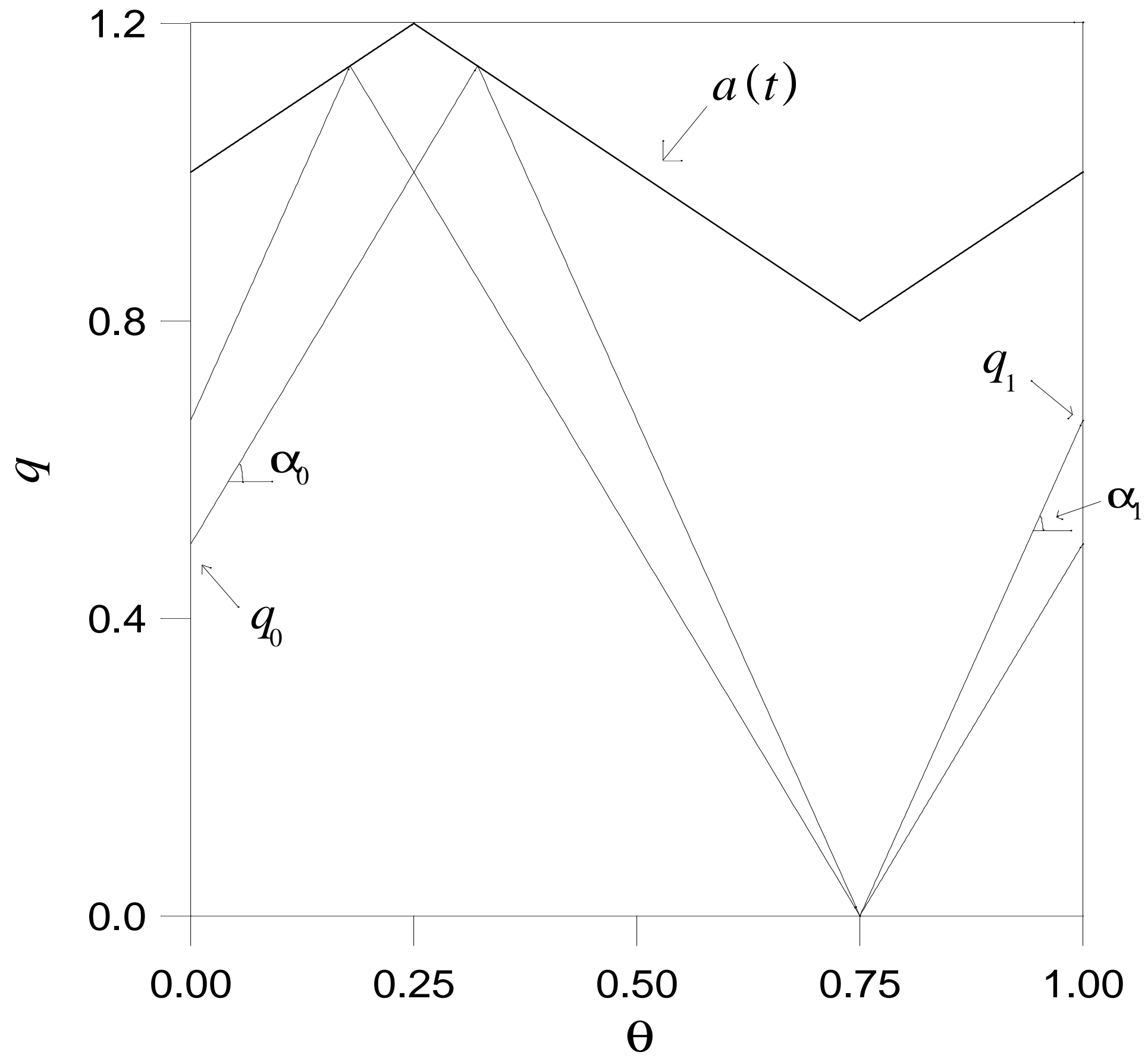




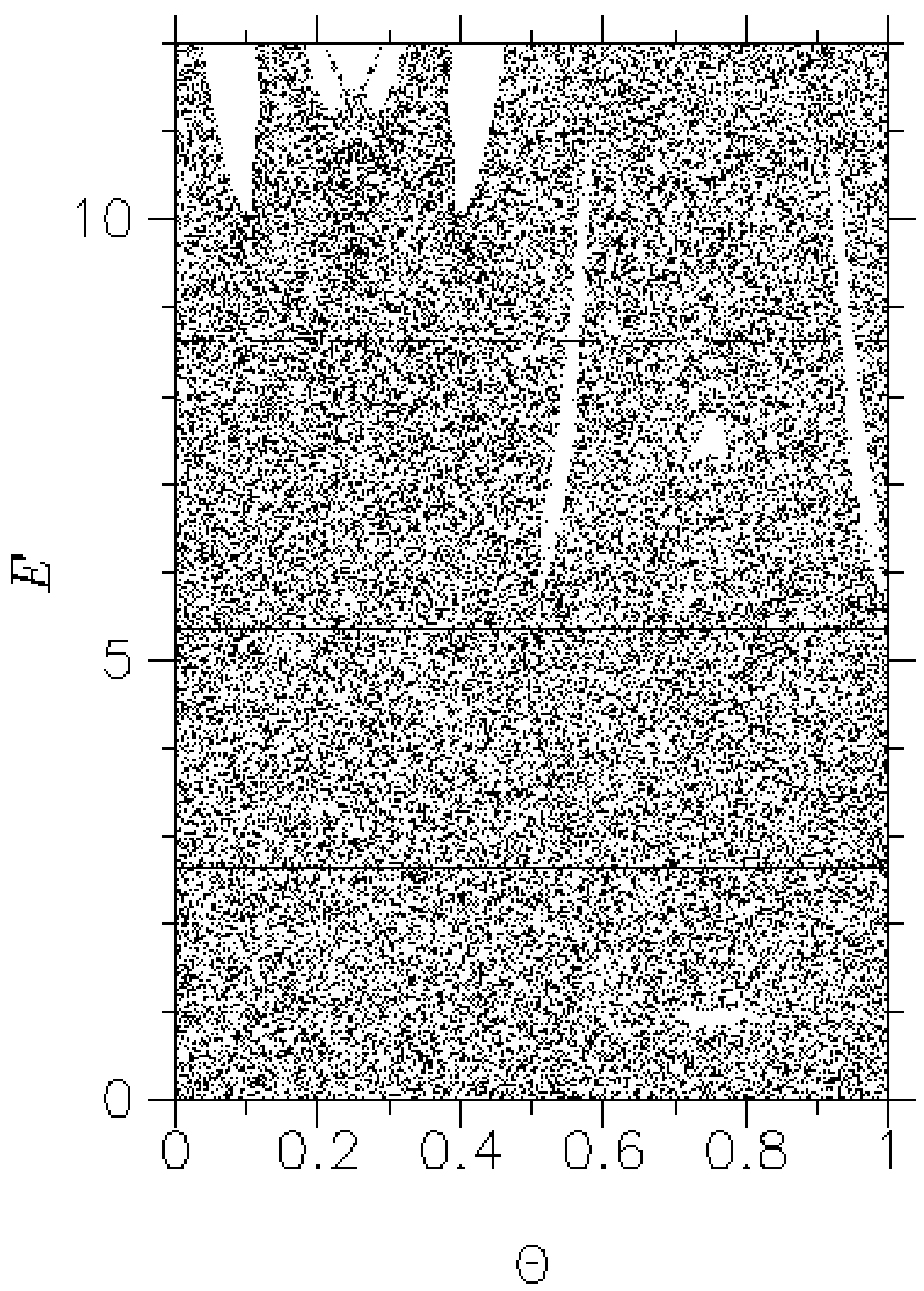




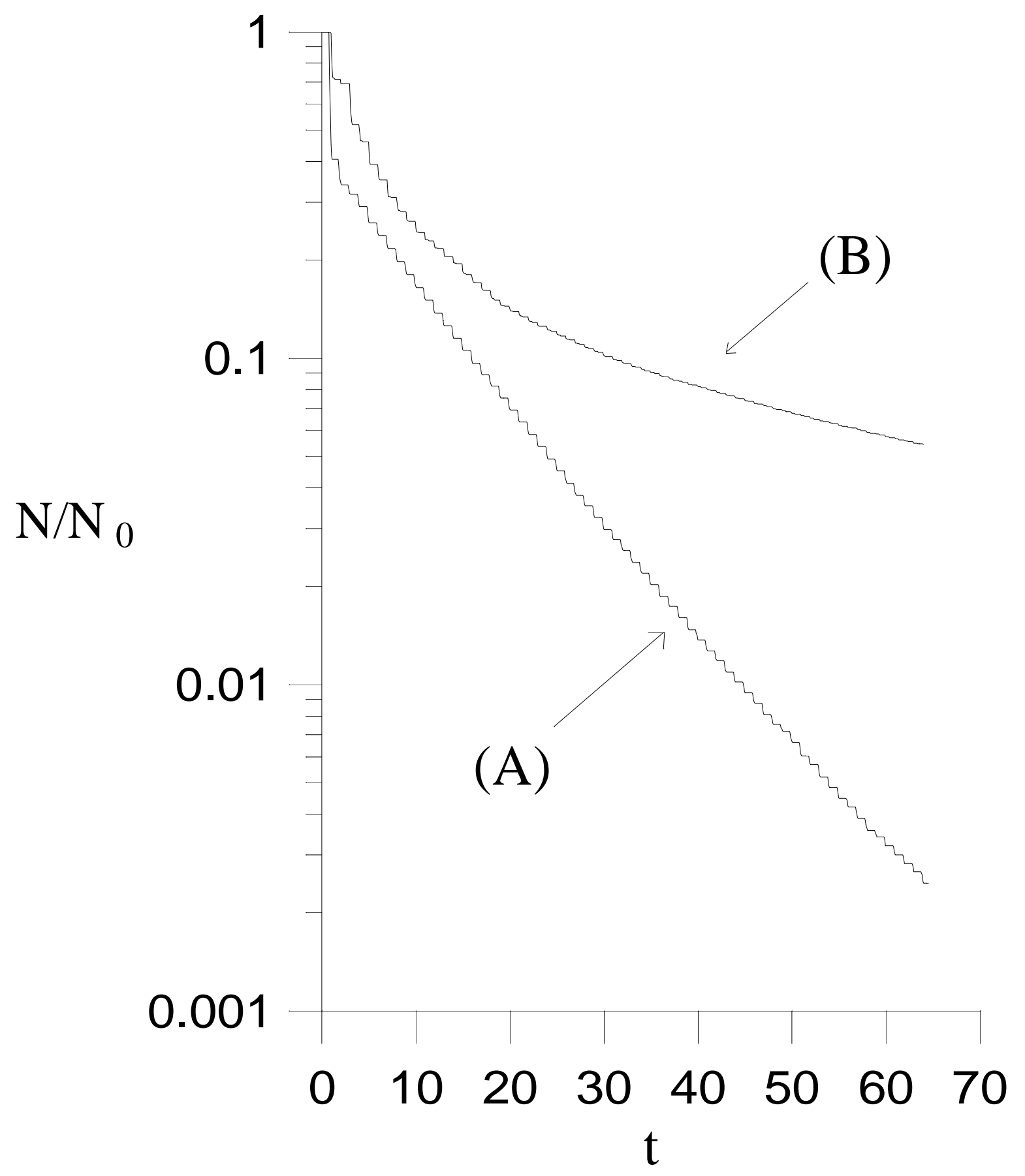

(a) 


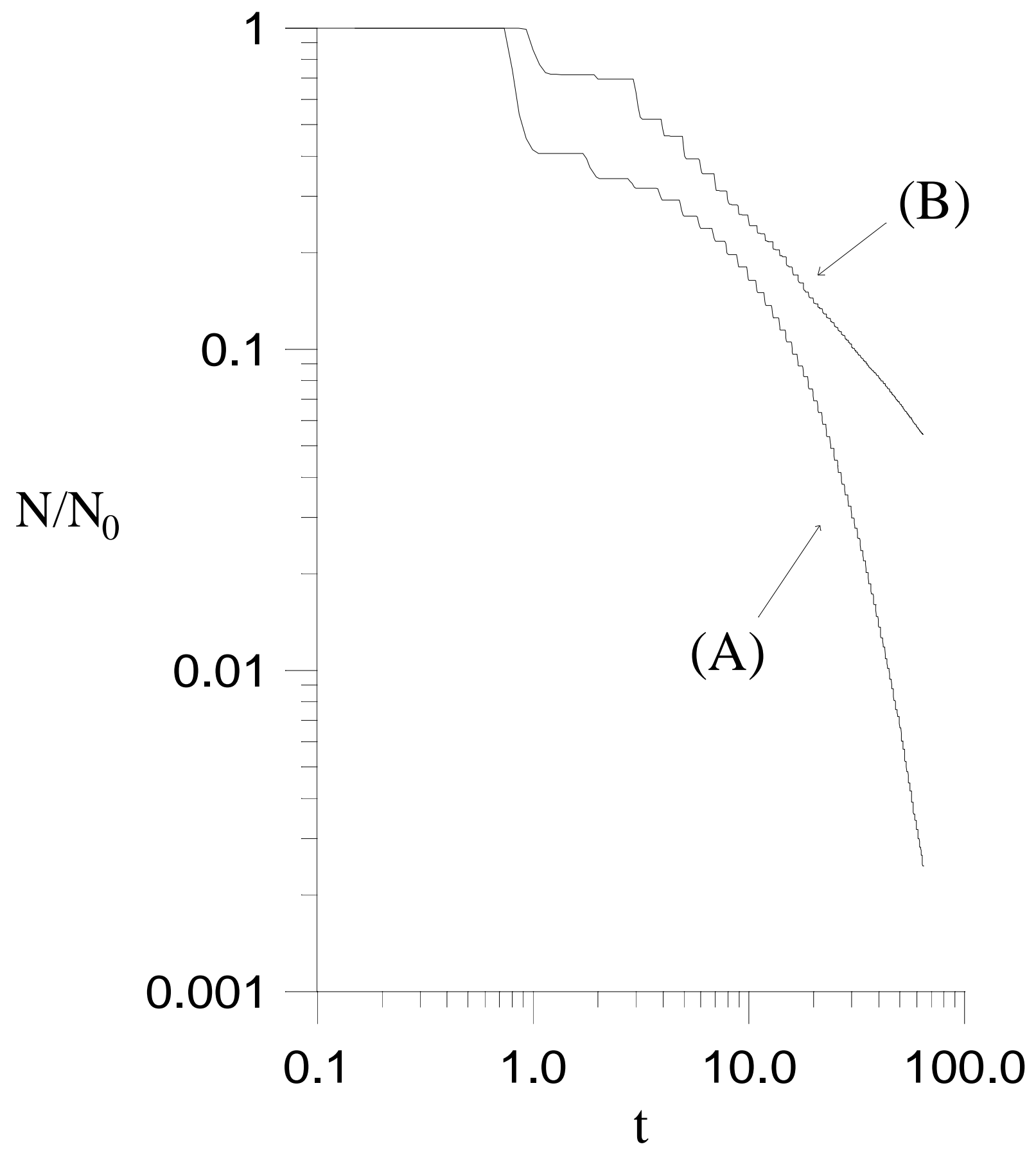

(b) 


$$
a(t)
$$

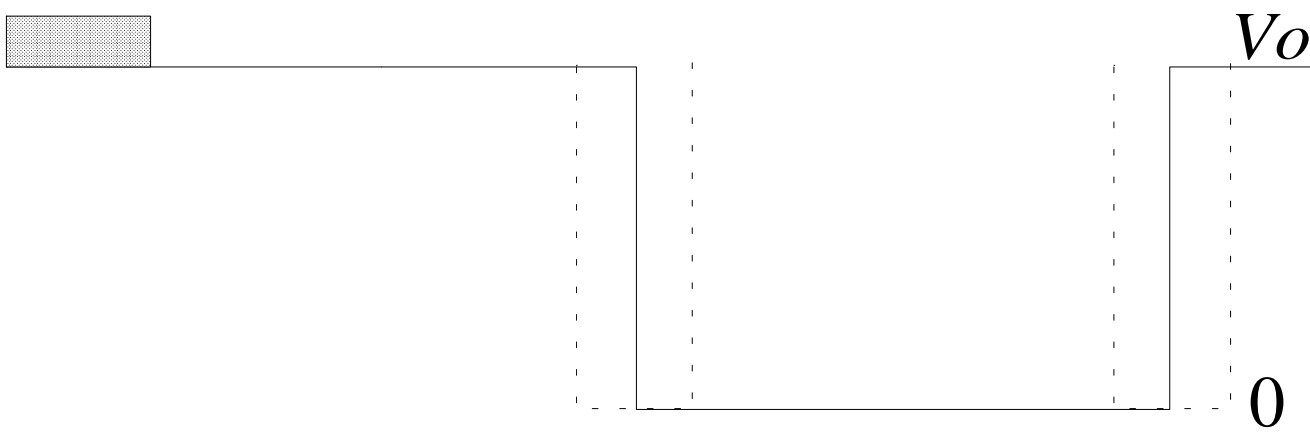

$$
L=1
$$




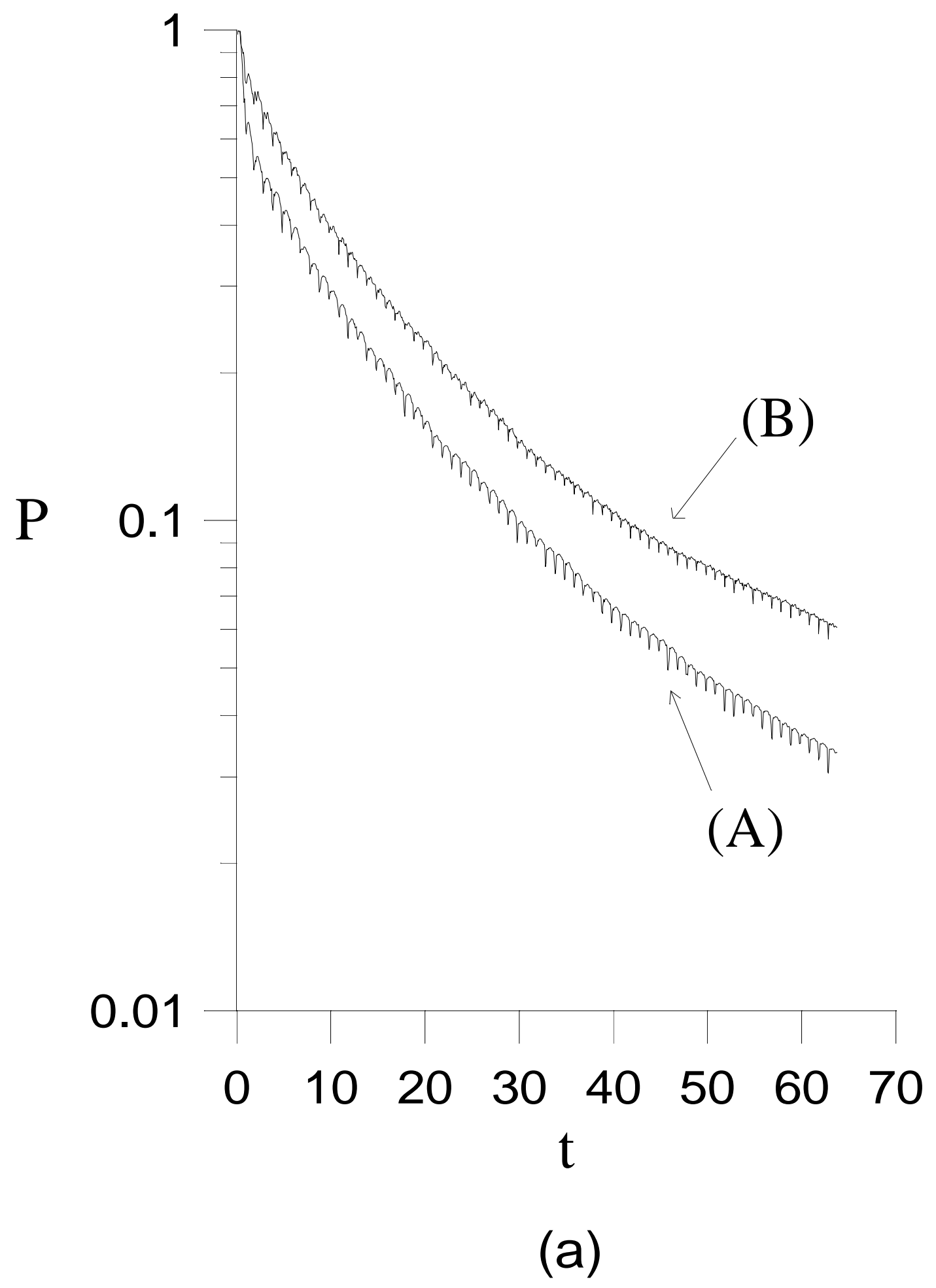




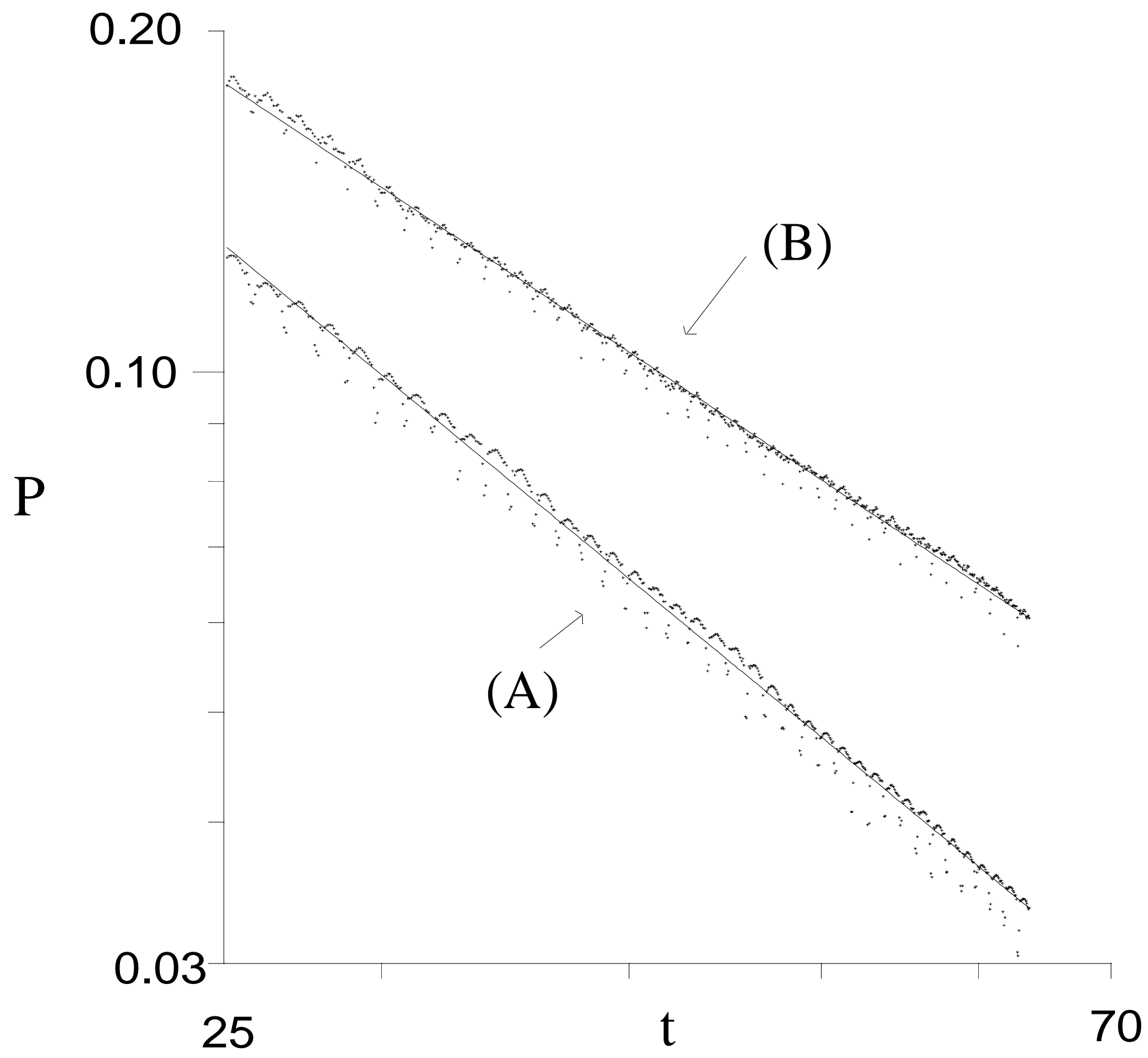

(b) 


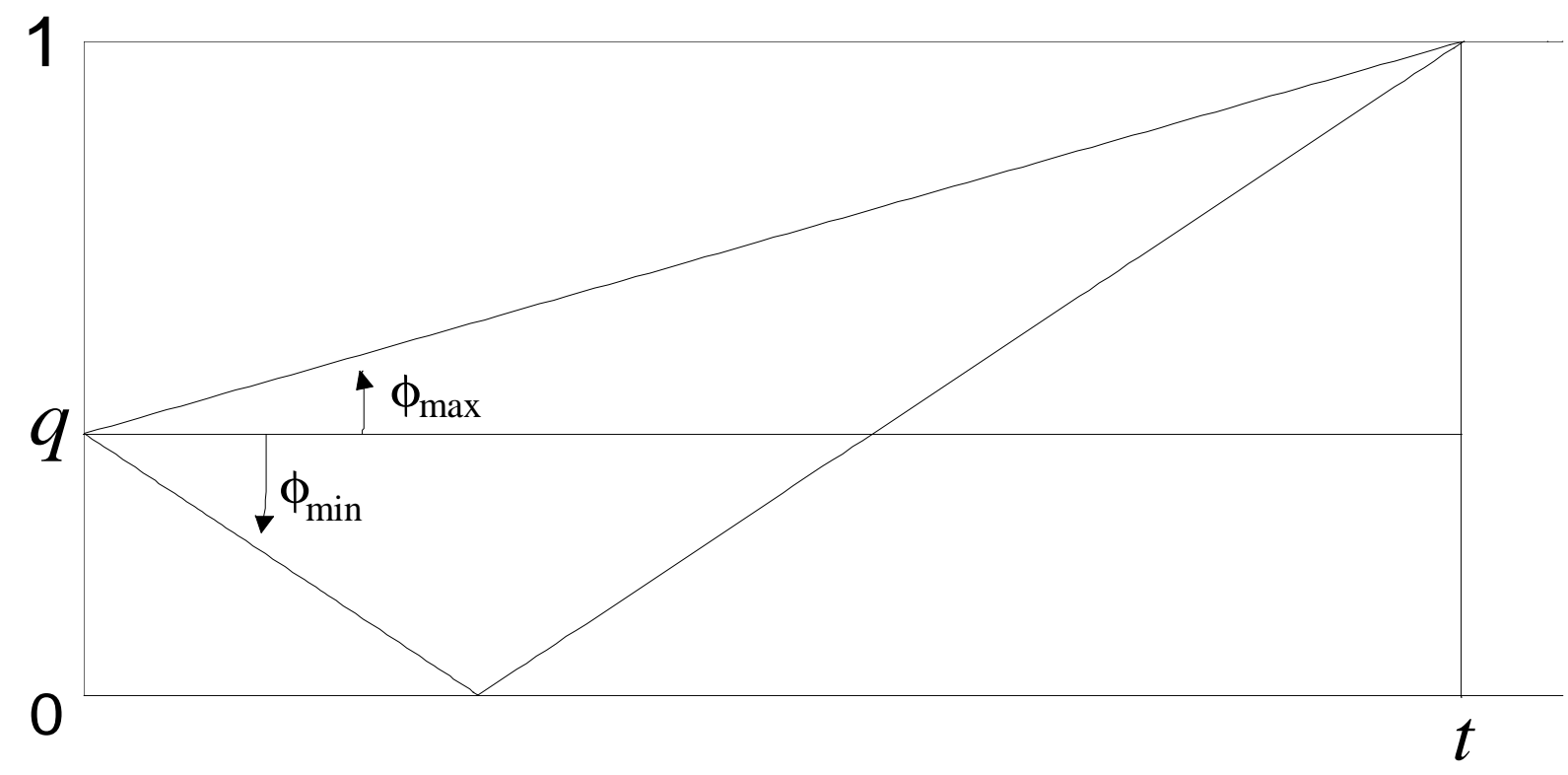




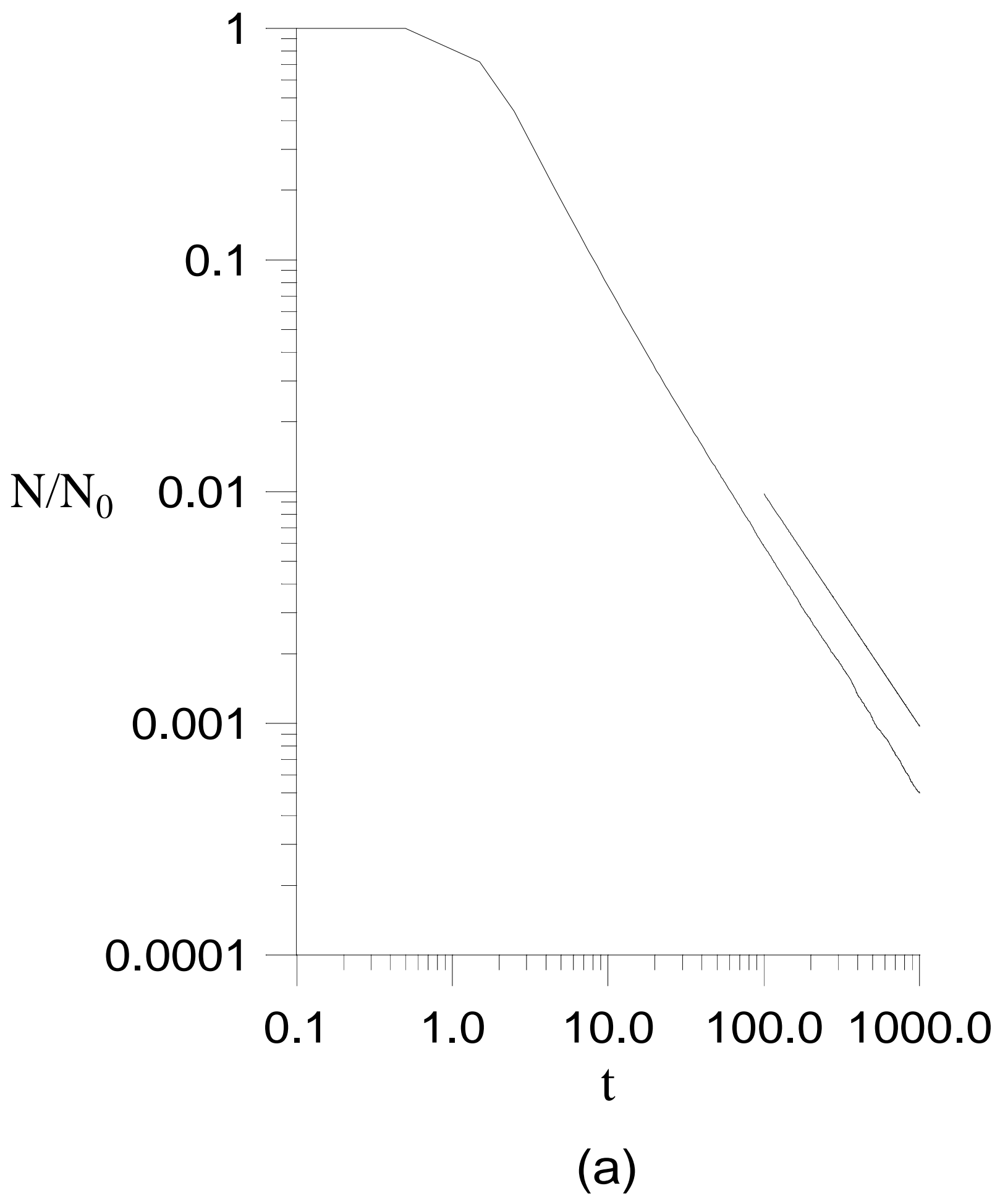




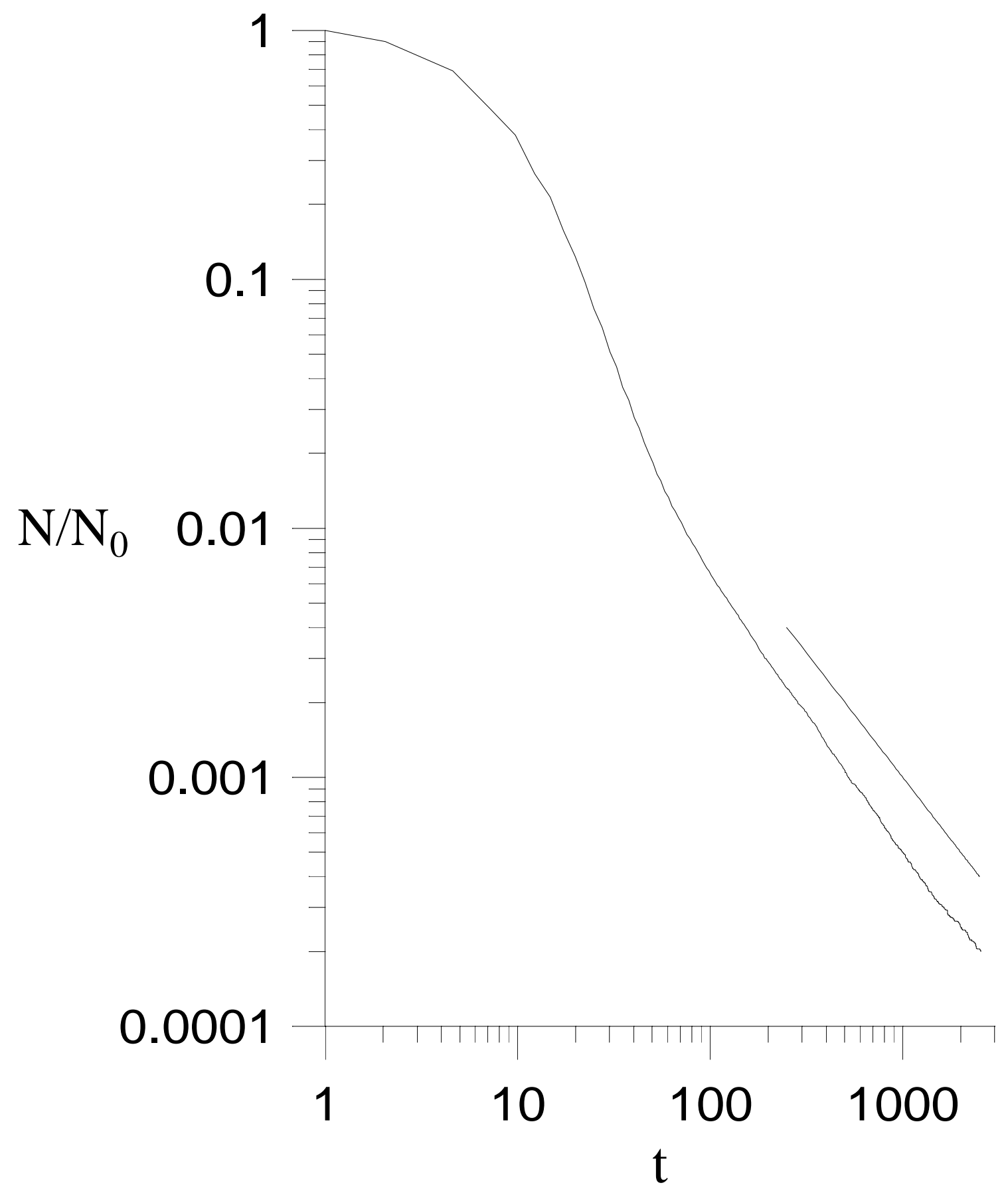

(b) 


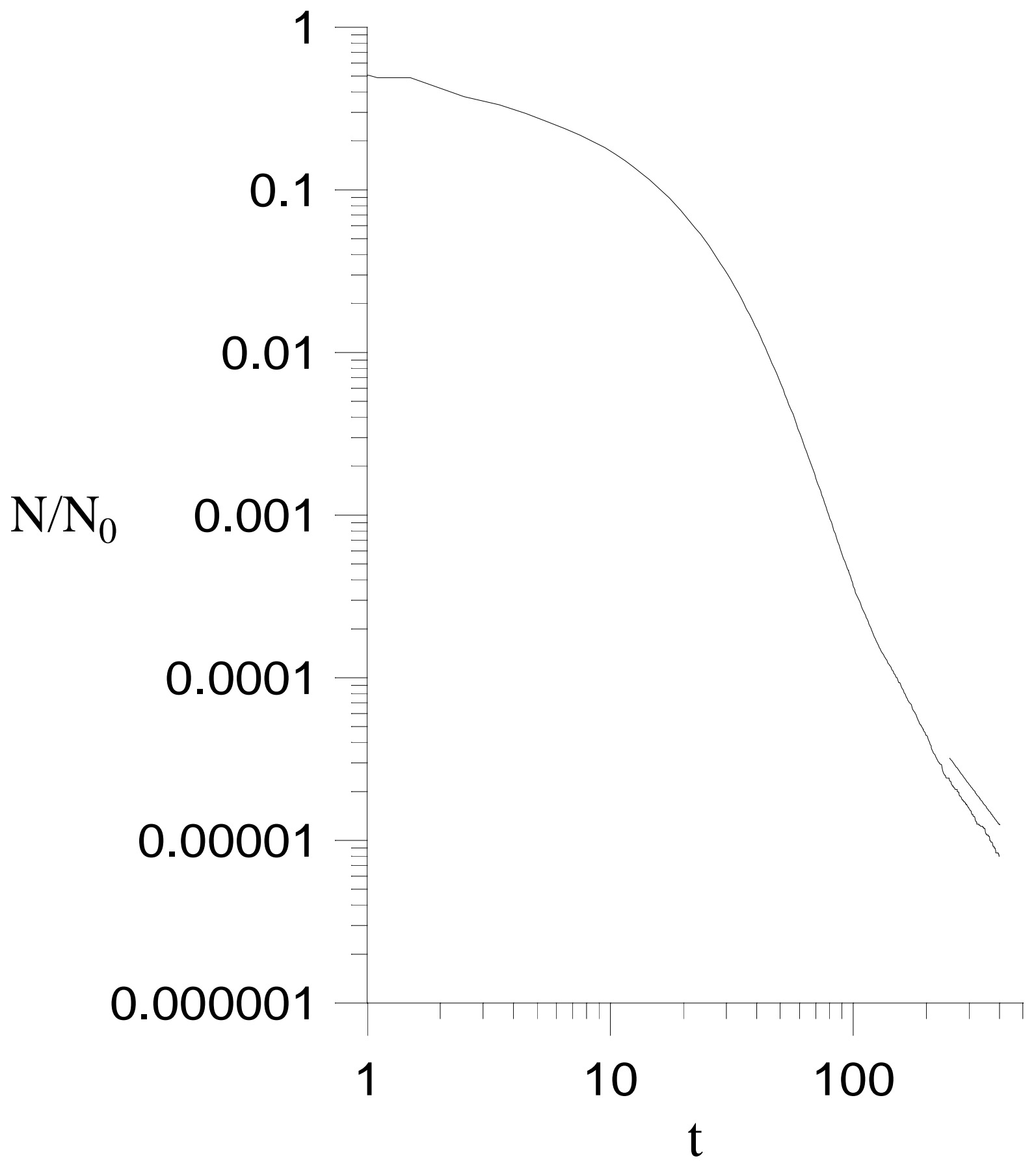

